Proyecciones

Vol. 20, No 2, pp. 139-175, August 2001.

Universidad Católica del Norte

Antofagasta - Chile

\title{
BOUNDS FOR CONFORMAL AUTOMORPHISMS OF RIEMANN SURFACES WITH CONDITION (A) *
}

\author{
RUBÉN A. HIDALGO \\ Universidad Técnica Federico Santa María, Chile
}

\begin{abstract}
In this note we consider a class of groups of conformal automorphisms of closed Riemann surfaces containing those which can be lifted to some Schottky uniformization. These groups are those which satisfy a necessary condition for the Schottky lifting property. We find that all these groups have upper bound $12(g-1)$, where $g \geq 2$ is the genus of the surface. We also describe a sequence of infinite genera $g_{1}<g_{2}<\cdots$ for which these upper bound is attained. Also lower bounds are found, for instance, (i) 4( $g+1)$ for even genus and $8(g-1)$ for odd genus. Also, for cyclic groups in such a family sharp upper bounds are given.
\end{abstract}

Subjclass : [2000] Primary 30F10, 30F40

Keywords : Schottky groups, Riemann surfaces, conformal automorphisms

*Partially supported by projects UTFSM 12.01.22, Fondecyt 1000715 and Fondecyt 1010093. 


\section{Introduction}

Retrosection theorem asserts that for each closed Riemann surface $S$, there are a Schottky group $G$, with region of discontinuity $\Omega$, and a Galois covering $P: \Omega \rightarrow S$ with $G$ as covering group. We say that $(\Omega, G, P: \Omega \rightarrow S)$ is a Schottky uniformization of $S$.

Assume that we are also given a group $H$ of conformal automorphisms of $S$. We may ask for the existence of a Schottky uniformization of $S$ for which the group $H$ lifts. If such an uniformization exists, then we say that $H$ is of Schottky type.

Is a trivial fact that each group $H$ of conformal automorphisms of the Riemann sphere is of Schottky type. In genus one, we have that the automorphisms of order three, four and six, having fixed points, cannot be lifted to any Schottky uniformization of genus one. Moreover, if $H$ does not contains any of these transformations, then it is of Schottky type [8].

In the case of genus greater or equal to two, the group $H$ is necessarily finite (the order is bounded by $84(g-1)$, the Hurwitz's bound). In [8] we have found a necessary condition, called condition (A), to be satisfied by $H$ in order for it to be of Schottky type.

In general, condition (A) is not sufficient for $H$ to be of Schottky type. In [10] there is an example of a group isomorphic to $\mathcal{S}_{5}$ acting on a surface of genus 56 satisfying condition (A) but not of Schottky type. In section 2 we show another example in genus four of a group of conformal automorphisms isomorphic to $\mathcal{S}_{5}$ which also satisfies condition (A) but not of Schottky type.

On the other hand, condition (A) turns out to be sufficient for abelian groups, dihedral groups, the alternating groups $\mathcal{A}_{4}, \mathcal{A}_{5}$ and the symmetric group $\mathcal{S}_{4}$ (see [7], [9] and [10]).

Groups satisfying automatically condition (A) are given by (i) embeddable groups, that is, the groups that can be embedded into three-dimensional Euclidean space as restrictions of rotations [18]; (ii) conformal groups acting free fixed points; (iii) dihedral groups; and (iv) finite groups of orientation-preserving homeomorphisms of the boundary of a handlebody which can be extended to the handlebody (Nielsen's realization problem [11] asserts that the Schottky type property is equivalent to the this extendability property (see also [15] and 
[19]).

A group $H$ of conformal automorphisms of a closed Riemann surface $S$ is called triangular if $S / H$ is the Riemann sphere with exactly three branch values. In section 2 (see proposition 1 ) we note that triangular groups (in genus greater or equal to two) cannot be of Schottky type. It is easy to see that cyclic triangular groups cannot satisfy condition (A). The example of section 2 is a triangular group, isomorphic the symmetric group in five letters, acting in genus four which satisfies condition (A) but not of Schottky type.

A group $H$ of conformal automorphisms which is not triangular (in genus $g \geq 2$ ) has order at most $12(g-1)$ as consequence of the Riemann-Hurwitz's formula [4]. In particular, this holds for a group of conformal automorphisms of Schottky type. We conjecture that a group satisfying condition (A) of order $12(g-1)$ must be of Schottky type. A first evidence of this is given in some way by theorem 6 .

In these notes we compute some bounds for non-triangular groups of conformal automorphisms of closed Riemann surfaces of genus $g \geq$ 2 , satisfying condition (A). The bounds obtained in this paper are the same as the ones obtained in [19], [16] and [15], for the class of conformal groups of Schottky type. It appears that groups satisfying condition (A) which are not of Schottky type are, in some sense, few in comparison to the ones which are of Schottky type.

The above permits us to conjecture that condition (A) is, in mostly of the cases, a sufficient condition for the group to be of Schottky type. A natural question is to determine which special properties have those groups for which condition (A) holds but are not of Schottky type. A discussion about this will appear elsewhere.

If the group is not triangular, satisfies condition (A) and its order is bigger or equal than $4(g-1)$, then we have that its order has the form $\frac{4 n}{(n-2)}(g-1)$, for some $n \geq 3$ (see corollary 6$)$. For $n=3$ we have the maximum order $12(g-1)$.

For abelian groups, satisfying condition (A) (equivalently, of Schottky type), we obtain that the order is at most $2(g+1)$ if $g \neq 5$ and 16 for $g=5$.

In section 5 we use similar arguments as in [12] to obtain an infinite sequence of positive integers $g_{1}=2, g_{2}=3, g_{3}=5, g_{4}=17, \ldots$, for 
which there is a group of automorphisms of Schottky type of order $12\left(g_{i}-1\right)$ acting on a Riemann surface of genus $g_{i}$.

In section 6 , we construct explicit pairs $(S, H)$, where $S$ is a closed Riemann surface of genus $g \geq 2$ and $H$ is a group of conformal automorphisms of $S$, of Schottky type and order:

(i) $4(g+1)$, every $g \geq 2$;

(ii) $8(g-1)$, if $g$ is odd;

(iii) $2(g+1)$ if $g$ is even (resp., $2 g$ if $g$ is odd) and $H$ a cyclic group;

(iv) 16 if $g=5$ and $H$ abelian group.

As a consequence of this, the minimal bound for groups satisfying condition $(\mathrm{A})$ is given by $4(g+1)$ (resp. $8(g-1))$ for $g$ even (resp. odd).

In order to connect this with the works of McCullough, Miller and Zimmermann, we have written section 7 . There we consider finite groups of orientation-preserving homeomorphisms of a handlebody of genus $g$. They correspond exactly to the finite groups of conformal automorphisms of some Riemann surface that can be lifted to some Schottky uniformization (see [19] and [11]). As a consequence of the results obtained in this note, we obtain again the bounds of [15], [16] and [19].

\section{Preliminaries and Condition (A)}

A group $H$ of conformal automorphisms of a Riemann surface $S$ is called triangular if the quotient surface $S / H$ has signature $(0,3 ; v, w, k)$, that is, it is a sphere with three singular points.

A purely loxodromic Kleinian group isomorphic to a free group of finite rank $k$ is called a Schottky group of genus $k$. We reefer to the papers [3], [13] and [14] for generalities on Schottky groups.

A Schottky uniformization of $S$ is a triple $(\Omega, G, \pi: \Omega \rightarrow S)$, where $G$ is a Schottky group with region of discontinuity $\Omega$ and $\pi: \Omega \rightarrow S$ is a regular holomorphic covering with $G$ as covering group. 
A Kleinian group $K$ containing a Schottky group $G$ as a subgroup of finite index is called a Kleinian finite extension of $G$. If $G$ is also normal in $K$ we call $K$ a Kleinian finite normal extension of $G$. In the above cases, the groups $K$ and $G$ have the same region of discontinuity (see $[13])$.

Kleinian finite normal extensions of Schottky groups appear in the following way. Let $S$ be a closed Riemann surface of genus $g$ and let $H$ be a group of conformal automorphisms (of finite order if $g \leq 1$ ). Assume there is a Schottky uniformization $(\Omega, G, \pi: \Omega \rightarrow S)$ of $S$, for which the group $H$ lifts as a group of conformal automorphisms of $\Omega(G)$ (we say that $H$ is of Schottky type).

Let us consider the group $K$ generated by the lifting of $H$. Clearly, the group $K$ contains $G$ as a normal subgroup of finite index. Since every conformal automorphisms of the region of discontinuity of a Schottky group is a Möbius transformation [1], the group $K$ is a Kleinian finite normal extension of the Schottky group $G$. From the above argument, we see that all Kleinian finite normal extensions of Schottky groups arise in this way. The following gives information about the elliptic transformations of $K$.

Theorem 1. Let $K$ be a Kleinian finite normal extension of a Schottky group $G$, and $\Omega$ the region of discontinuity of them. If $h \in K$ is an elliptic transformation, then either both fixed points of $h$ belong to $\Omega$ or there exists a loxodromic element $g \in G$ commuting with $h$.

The proof can be found in [8]. Before to write condition (A), let us recall how it appears. Let us consider a closed Riemann surface $S$ of genus $g \geq 2$ and a group $H$ of conformal automorphisms of $S$ of Schottky type. Let $(\Omega, G, \pi: \Omega \rightarrow S)$ be a Schottky uniformization of the surface $S$ such that every element of $H$ can be lifted to a conformal automorphism of $\Omega$. Denote by $K$ the group generated by the liftings of the elements of $H$ to $\Omega$ by $\pi: \Omega \rightarrow S$. The group $K$ is a Kleinian finite normal extension of $G$ with index equal to the order of $H$.

For a point $p \in S$, we define the stabilizer of $p$ in $H$ as the subgroup $H(p)$ of $H$ consisting of those elements that fix the point $p$. Generically, $H(p)$ consists only of the identity element.

For $h \in H(p)$, of order greater than 2 ; let $(U, z)$ any chart of $S$ such that $p$ belongs to $U$ and $z(p)=0$. In this chart $h$ looks locally 
as the map $z \rightarrow e^{2 i \theta}$, where $\theta$ is well defined modulo $2 \pi$. We choose $\alpha(h, p)$ to be the representative of the above $\theta$ in the open interval $(-\pi, \pi)$. This definition is independent of the choice of chart. We call this number the rotation number of $h$ at $p$. Now, as a consequence of Theorem 1 we obtain the following property, called condition (A), to be satisfy by the group $H$.

\section{Condition (A)}

The set of fixed points of the non-trivial elements of $H$ can be put into pairs satisfying the following properties.

(A1) If $\{p, q\}$ is such a pair, then $p \neq q, H(p)=H(q)$ and $\alpha(h, p)=$ $-\alpha(h, q)$, for $h \in H(p)=H(q)$ of order greater than two.

(A2) If $\{p, q\}$ and $\{r, t\}$ are two such pairs, then either $\{p, q\} \cap\{r, t\}=$ $\emptyset$ or $\{p, q\}=\{r, t\}$.

(A3) If $\{p, q\}$ is a pair and $t \in H$ is so that $t(p)=q$, then $t$ has order two.

(A4) If $p$ is fixed point of some non-trivial element of $H$, then there is another fixed point $q$ so that $\{p, q\}$ is one of the above pairs.

\section{Condition $\left(\mathrm{A}^{*}\right)$}

This is the same as condition (A), but replacing (A3) by $\left(\mathrm{A} 3^{*}\right)$ for each pair $\{p, q\}$ there is no transformation $t \in H$ so that $t(p)=q$.

\section{Remarks.}

(1) If the order of $H$ is odd, then condition (A) is equivalent to condition $\left(\mathrm{A}^{*}\right)$.

(2) If the group $H$ is cyclic, then part (A3) cannot happen. In particular, $H$ cannot be a cyclic triangular group satisfying condition (A).

(3) If $H$ is abelian and there is a pair $\{p, q\}$ permuted by some involution, then $H(p)=H(q)$ is the group in two elements. 
(4) It is not difficult to assume the property that if $\{p, q\}$ is a pair, then for every $h \in H$ we have that $\{h(p), h(q)\}$ is also one of our pairs.

(5) If the group $H$ acts freely or it is isomorphic to a dihedral group, then it satisfies trivially condition (A).

(6) If $H$ is a group of conformal automorphisms satisfying condition (A), then every subgroup $K<H$ also satisfies it.

From now on, we assume the pairing to satisfy part (3) of the above remark. We say that two pairs $\{p, q\}$ and $\{r, t\}$ are equivalent under $H$ if there is an element $h \in H$ such that $\{h(p), h(q)\}=\{r, t\}$.

\section{Triangular Groups and Schottky Type Prop- erty}

In this section, we observe that a triangular group cannot be of Schottky type. More precisely,

Proposition 1. Let $S$ be a closed Riemann surface of genus $g \geq 2$, and $H$ be a triangular group of conformal automorphisms of $S$. Then there is no Schottky uniformization of $S$ for which the group $H$ lifts.

Proof. Let us assume there is a Schottky uniformization $(\Omega, G, \pi$ : $\Omega \rightarrow S$ ) of $S$ for which the group $H$ lifts. Consider the group $K$, generated by $G$ and the lifts of $H$. As observed before, the group $K$ is geometrically finite Kleinian group with $\Omega$ as region of discontinuity, and without parabolic elements. As a consequence (see the work of Keen, Maskit and Series in [5]), we have that $K$ is a totally parabolic Kleinian group and, in particular, the connected components of $\Omega$ are round discs. Since the region of discontinuity of a Schottky group is connected, we have that $\Omega$ is just a round disc, a contradiction.

A natural question, after the above result, is the following: Is there some triangular group (for genus greater or equal to two) satisfying condition $(\mathrm{A})$ ? It is clear that they cannot satisfy condition $\left(\mathrm{A}^{*}\right)$.

In genus four there is an example of a closed Riemann surface with a group $H$ of automorphisms, isomorphic to $\mathcal{S}_{5}$, which is triangular and 
satisfies condition (A). Such an example [6] is given by the algebraic curve in $\mathbf{C} P_{3}$ defined by

$$
z_{1} z_{4}+z_{2} z_{3}=0 \quad z_{1}^{2} z_{3}+z_{1} z_{2}^{2}+z_{3}^{2} z_{4}+z_{2} z_{4}^{2}=0,
$$

where the group has generators

$$
A=\left(\begin{array}{rrlr}
1 & 0 & 0 & 0 \\
0 & w & 0 & 0 \\
0 & 0 & w^{2} & 0 \\
0 & 0 & 0 & w^{3}
\end{array}\right)
$$

and

$$
B=\left(\begin{array}{lrlr}
w^{2}+w^{3} & -w^{3} & w & -1-w^{3} \\
-w^{2} & 1+w^{2}+w^{3} & 1+w & w \\
w^{4} & 1+w^{4} & 1+w^{2}+w^{3} & -w^{3} \\
-1-w^{2} & w^{4} & -w^{2} & w^{2}+w^{3}
\end{array}\right)
$$

where $w$ is a quintic primitive square root of unity.

The surface $S / H$ is the Riemann sphere branched at three points with branching 2,4 and 5 , respectively. The subgroups of $H$, acting with fixed points, are given by:

(i) Cyclic groups $K$ of order 4 , acting with two fixed points, so that $S / K$ is a genus one surface branched at two points (with branching 4 ) and $K$ satisfies condition (A);

(ii) Cyclic groups $K$ of order 5 acting with four fixed points, with $S / K$ a genus zero branched at four points with branching 5 and $K$ satisfying condition (A); and

(iii) Cyclic groups of order $6 K$ so that the only tranformation with fixed points is given by the element of order two. The quotient $S / K$ is a genus one surface branched at two points with branching 2 and, in paticular satisfying condition (A);

It follows from the above that the triangular group $H$ satisfy condition (A). For cyclic triangular groups, part (2) of the remark at the end of section 1 asserts that they cannot satisfy condition (A). 
Corollary 1. Let $S$ be a closed Riemann surface of genus $g \geq 2$ and $f: S \rightarrow S$ be a conformal automorphism of order $N$. If $N>2(g+1)$ then the cyclic group generated by the automorphism $f$ cannot satisfy the condition (A) and, in particular, cannot be lifted to any Schottky covering of $S$.

Proof. It is known (see [17]) that if $N>2(g+1)$, then the quotient Riemann surface $S /<f>$ has signature $(0,3 ; v, w, k)$. As remarked before, a cyclic group satisfying condition (A) cannot be triangular.

Compare Corollary 1 to Theorems 3 and 4 . If $g=3$, then Corollary 1 asserts that cyclic groups of order greater than 8 cannot be lifted to Schottky coverings. In this genus there is exactly one class of non-hyperelliptic Riemann surfaces admitting an automorphism of order 7 and, for this automorphism, the quotient surface is the sphere with three branch points of order 7 . Proposition 1 implies that this automorphism of order seven cannot be lifted to any Schottky covering.

\section{The Canonical Homomorphism of Condition (A)}

Let $S$ and $H$ be a closed Riemann surface of genus $g \geq 2$ and a group of conformal automorphisms satisfying condition (A), respectively. Consider the natural holomorphic branched regular covering $\Pi: S \rightarrow S / H$ induced by the action of $H$ on $S$. The branch locus $\mathcal{B}$ of this covering corresponds to the projection of the fixed points of non-trivial elements of $H$.

Let $\left\{p_{i}, q_{i}\right\},\left\{r_{j}, t_{j}\right\}, i=1, \ldots, k_{1}, j=1, \ldots, k_{2}$, be a maximal set of non-equivalent pairs under $H$ such that, the orbit under $H$ of $p_{i}$ does not contain $q_{i}$ and the orbit under $H$ of $r_{j}$ contains $t_{j}$. Let us denote by $v_{i}$ the order of the group $H\left(p_{i}\right)$ and by $w_{j}$ the order of $H\left(r_{j}\right)$.

The surface $S / H$ has signature (see [4]) of the form

$$
\left(\gamma, 2 k_{1}+k_{2} ; v_{1}, v_{1}, \ldots, v_{k_{1}}, v_{k_{1}}, w_{1}, \ldots, w_{k_{2}}\right) .
$$

Remark. If the group $H$ is cyclic, then $k_{2}=0$. If $H$ is abelian and $k_{2} \neq 0$, then $w_{j}=2$. 
If $N$ denotes the order of the group $H$, then the Riemann-Hurwitz's formula [4] implies the equality

$$
2(g-1)=N\left(2(\gamma-1)+2 \sum_{i=1}^{k_{1}}\left(1-1 / v_{i}\right)+\sum_{j=1}^{k_{2}}\left(1-1 / w_{j}\right)\right) .
$$

If we denote by $\left.J=2(\gamma-1)+2 \sum_{i=1}^{k_{1}}\left(1-1 / v_{i}\right)+\sum_{j=1}^{k_{2}}\left(1-1 / w_{j}\right)\right)$, then $J>0$ (since $g>1$ ) and $N$ is maximal for $J$ minimal.

Let us denote by $X$ the Riemann surface $S / H-\mathcal{B}$ and by $S^{\prime}$ the Riemann surface $S$ minus the fixed points of the non-trivial elements of $H$. Consider the unbranched regular covering $\Pi: S^{\prime} \rightarrow X$ obtained by restriction of $\Pi: S \rightarrow S / H$. Let $x$ be any point in $X$ and consider oriented simple loops, based at $x$, say $\alpha_{n}, \beta_{n}, \delta_{i}, \eta_{i}$ and $\theta_{j}, n=1, \ldots, \gamma$, $i=1, \ldots, k_{1}$ and $j=1, \ldots, k_{2}$, satisfying the following properties (see figure 1).

(1) The loop $\theta_{j}$ is free homotopic to a simple loop around the puncture determined by $\Pi\left(r_{j}\right)$, and it is oriented such that the puncture is at the left side.

(2) The loop $\delta_{i}$ is free homotopic to a simple loop around the puncture determined by $\Pi\left(p_{i}\right)$, and it is oriented such that the puncture is at the left side.

(3) The loop $\eta_{i}$ is free homotopic to a simple loop around the puncture determined by $\Pi\left(q_{i}\right)$, and it is oriented such that the puncture is at the left side.

(4) The loops $\alpha_{n}$ and $\beta_{n}$ form a canonical basis for the surface $S$, and oriented such that the intersection number of $\alpha_{i}$ and $\beta_{i}$ (in this order) is +1 .

The above set of loops is a basis for the fundamental group of $X$ with the following presentation

$$
\Pi_{1}(X, x)=<\alpha_{n}, \beta_{n}, \delta_{i}, \eta_{i}, \theta_{j} ; \Pi_{n=1}^{\gamma}\left[\alpha_{n}, \beta_{n}\right] \Pi_{i=1}^{k_{1}} \delta_{i} \Pi_{i=1}^{k_{1}} \eta_{i} \Pi_{j=1}^{k_{2}} \theta_{j}=1>,
$$

where $[a, b]$ is the commutator of $a$ and $b$, that is, $a b a^{-1} b^{-1}$. This is a free group if and only if $k_{1}+k_{2}>0$.

We have a canonical surjective homomorphism $\Phi: \Pi_{1}(X, x) \rightarrow H$, induced by the action of $H$ on $S$. In the kernel of this homomorphism we have the elements $\delta_{i} \eta_{i}, \delta_{i}^{v_{i}}$ and $\theta_{j}^{w_{j}}$. 


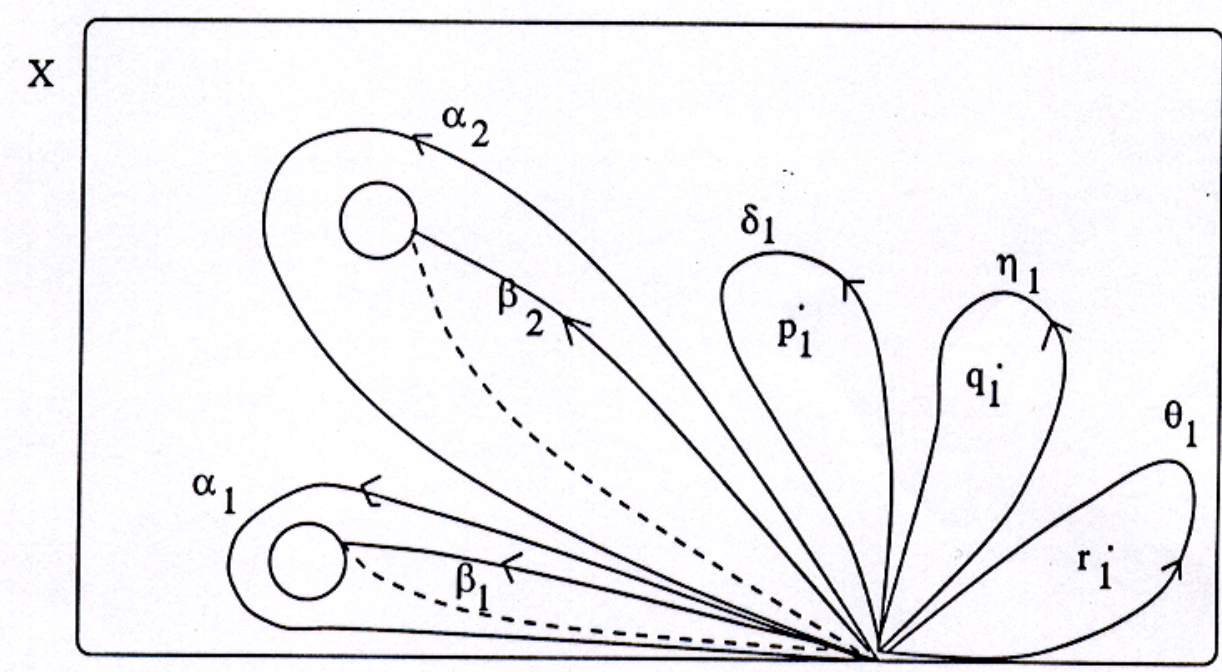

FIGURE 1. $\gamma=2, k_{1}=1$ and $k_{2}=1$

\section{Bounds for Conformal Groups with Condition}

(A)

In this section we consider a closed Riemann surfaces $S$ of genus $g \geq 2$ and a group $H$ of conformal automorphisms of $S$ satisfying condition (A). Moreover, we assume that $S / H$ has no signature of type $(0,3 ; p, q, r)$, that is, $H$ is not triangular. By Riemann-Hurwitz's formula, it is direct to see that the order of $H$ is at most $12(g-1)$. If $H$ is abelian, then its order is at most $2(g+1)$. If $H$ is cyclic, then its order is at most $2 g$ (resp. $2(g+1))$ if $g$ is odd (resp. even). If the order of $H$ is odd, then it is at most $3(g-1)$. If $H$ does not contain non-abelian Dihedral subgroups, then its order is at most $6(g-1)$. The same situation occurs for the subclass of groups that have the lifting property [15].

Theorem 2. Let $S$ be a closed Riemann surface of genus $g \geq 2$. Let $H$ be a non-triangular group of conformal automorphisms of $S$ satisfying condition (A). If the order of $H$ is greater than $8(g-1)$, then its order is $12(g-1)$. Moreover, if $H$ satisfies condition $\left(A^{*}\right)$, then the order of $H$ is at most $6(g-1)$. 
Corollary 2. Let $K$ be a Kleinian finite normal extension of a Schottky group $G$ of genus $g \geq 2$. Then the index of $G$ in $K$ is at most $12(g-1)$. Moreover, if the index is greater than $8(g-1)$, then it is exactly $12(g-1)$.

A consequence of Theorem 2 together example 6.3 of section 6 is the following (see also [16]).

Corollary 3. The maximal index for a Kleinian finite normal extension of a Schottky group of genus $g \geq 2$, for $g$ odd, is either $8(g-1)$ or $12(g-1)$.

Remark. In [15] has been shown that: if $g=2 p+1, p>23$ a prime, then the maximal index of a Kleinian finite normal extension of a Schottky group of genus $g$ is $8(g-1)$.

Proof. (Theorem 2.) Let us assume that $H$ satisfy condition (A) and it is not triangular. By Riemann-Hurwitz's formula, to find the maximal values of the order of $H$, we need to find the minimal values of $\left.J=2 \gamma-2+2 \Sigma_{i=1}^{k_{1}}\left(1-1 / v_{i}\right)+\sum_{j=1}^{k_{2}}\left(1-1 / w_{j}\right)\right)$.

(A) Case $\gamma \geq 2$. In this case $J \geq 2$ and $N \leq g-1$.

(B) Case $\gamma=1$. In this case, $\left.J=2 \sum_{i=1}^{k_{1}}\left(1-1 / v_{i}\right)+\sum_{j=1}^{k_{2}}\left(1-1 / w_{j}\right)\right)$. Since $J>0$, then $k_{1}+k_{2} \geq 1$.

(B.1) If $k_{1}+k_{2}=1$, then $J=2\left(1-1 / v_{1}\right) \geq 1$ or $J=1-1 / w_{1} \geq 1 / 2$. In the first case $N \leq 2(g-1)$ and in the second case $N \leq 4(g-1)$.

(B.2) If $k_{1}+k_{2} \geq 2$, then $J=2 k_{1}+k_{2}-\left(2 \sum_{i=1}^{k_{1}} 1 / v_{i}+\sum_{j=1}^{k_{2}} 1 / w_{j}\right)$. We need to find the maximal value of $\left(2 \sum_{i=1}^{k_{1}} 1 / v_{i}+\sum_{j=1}^{k_{2}} 1 / w_{j}\right) \geq$ $2 k_{1} / 2+k_{2} / 2=k_{1}+k_{2} / 2 \geq 1$. So, $N \leq 2(g-1)$.

(C) Case $\gamma=0$. In this case $J=-2+2 \sum_{i=1}^{k_{1}}\left(1-1 / v_{i}\right)+\sum_{j=1}^{k_{2}}(1-$ $\left.1 / w_{j}\right)=\left(2 k_{1}+k_{2}-2\right)-\left(2 \sum_{i=1}^{k_{1}} 1 / v_{i}+\sum_{j=1}^{k_{2}} 1 / w_{j}\right)$. By hypotheses, $2 k_{1}+k_{2}>3$.

(C.1) If $2 k_{1}+k_{2} \geq 6$, then $J \geq 2 k_{1}+k_{2}-2-2 k_{1} / 2-k_{2} / 2=k_{1}+$ $k_{2} / 2-2 \geq 3-2=1$. So, $N \leq 2(g-1)$. 
(C.2) If $2 k_{1}+k_{2}=5$, then the only possible pairs $\left(k_{1}, k_{2}\right)$ are $(0,5)$, $(1,3)$ or $(2,1)$.

(1) If $k_{1}=0$ and $k_{2}=5$, then $J=5-2-\Sigma_{j=1}^{5} 1 / w_{j} \geq 3-5 / 2=$ $1 / 2$. So, $N \leq 4(g-1)$.

(2) If $k_{1}=1$ and $k_{2}=3$, then $J=2+3-2-2 / v_{1}-\Sigma_{j=1}^{3} 1 / w_{j} \geq$ $3-1-3 / 2=1 / 2$. So, $N \leq 4(g-1)$.

(3) If $k_{1}=2$ and $k_{2}=1$, then $J=4+1-2-2 \Sigma_{i=1}^{2} 1 / v_{i}-1 / w_{1} \geq$ $3-2-1 / 2=1 / 2$. So, $N \leq 4(g-1)$.

(C.3) If $2 k_{1}+k_{2}=4$, then the possible pairs for $\left(k_{1}, k_{2}\right)$ are $(0,4),(1,2)$ or $(2,0)$.

(1) If $k_{1}=0$ and $k_{2}=4$, then $J=4-2-\Sigma_{j=1}^{4} 1 / w_{j}=2-$ $\sum_{j=1}^{4} 1 / w_{j} \geq 2-(1 / 2+1 / 2+1 / 2+1 / 3)=1 / 6$. So, $N \leq 12(g-1)$.

(2) If $k_{1}=1$ and $k_{2}=2$, then $J=2+2-2-2 / v_{1}-\Sigma_{j=1}^{2} 1 / w_{j}=$ $2-2 / v_{1}-\Sigma_{j=1}^{2} 1 / w_{j} \geq 2-1-1 / 2-1 / 3=1 / 6$. So $N \leq 12(g-1)$.

(3) If $k_{1}=2$ and $k_{2}=0$, then $J=4-2-2 \Sigma_{i=1}^{2} 1 / v_{i}=2-$ $2 \Sigma_{i=1}^{2} 1 / v_{i} \geq 2-2(1 / 2+1 / 3)=1 / 3$. So, $N \leq 6(g-1)$.

The largest of the above bounds for the order of $H$ is $12(g-1)$. Let us assume the order of $H$ to be greater than $8(g-1)$. From the above computations, we only need to take care of cases (C.3)-(1) and (C.3)-(2). In the first case, we have

$$
2(g-1)=N\left(2-\Sigma_{j=1}^{4} 1 / w_{j}\right)>8(g-1)\left(2-\Sigma_{j=1}^{4} 1 / w_{j}\right),
$$

that is, $\sum_{j=1}^{4} 1 / w_{j}>7 / 4$. We may assume $2 \leq w_{1} \leq w_{2} \leq w_{3} \leq w_{4}<$ $\infty$. The only 4 -tuples that satisfy the above inequality are $(2,2,2,2)$ and $(2,2,2,3)$. The first 4 -tuple implies $g=1$, a contradiction. The second 4 -tuple implies $N=12(g-1)$ in which case we are done. In the second case, we have

$2(g-1)=N\left(2-2 / v_{1}-\Sigma_{j=1}^{2} 1 / w_{j}\right)>8(g-1)\left(2-2 / v_{1}-\Sigma_{j=1}^{2} 1 / w_{j}\right)$, that is, $2 / v_{1}+\Sigma_{j=1}^{2} 1 / w_{j}>7 / 4$. If we assume $w_{1} \leq w_{2}$, then the only 3 -tuples $\left(v_{1}, w_{1}, w_{2}\right)$ satisfying the above inequality are $(2,2,2)$ and $(2,2,3)$. In the first one, we have $g=1$, a contradiction. The second 3 -tuple implies $N \leq 2$. In fact, consider the surjective homomorphism (of section 3 ) 


$$
\Phi: \Pi_{1}(X, x) \rightarrow H
$$

In this case, $\Pi_{1}(X, x)$ is generated by $\delta_{1}, \eta_{1}, \theta_{1}$ and $\theta_{2}$ with the only relation $\delta_{1} \eta_{1} \theta_{1} \theta_{2}=1$. The kernel of the above homomorphism is a normal subgroup containing the elements $\delta_{1} \eta_{1}, \delta_{1}^{2}, \theta_{1}^{2}$ and $\theta_{2}^{3}$. Consider the smallest normal subgroup $T$ of $\Pi_{1}(X, x)$ containing the above elements and let us consider the group $\Pi_{1}(X, x) / T$. The order of this group is greater than $N$. It is easy to see that this group is $\mathbf{Z} / 2 \mathbf{Z}$.

In the case that $H$ satisfies condition $\left(\mathrm{A}^{*}\right)$, we have case (C.3) part (3).

Corollary 4. Let $S$ and $H$ be as in Theorem 2. Then

(1) If $H$ does not contain non-abelian dihedral subgroups, then $N \leq$ $6(g-1)$.

(2) If $N$ is odd, then $N \leq 3(g-1)$.

Proof. (1) We only need to take care of case (C.3). In that case, we have three possibilities for the pair $\left(k_{1}, k_{2}\right)$. The no existence of nonabelian Dihedral subgroups of $H$ implies necessarily that $w_{j}=2$, for all $j$. For the pair $(0,4)$ it will imply that $J=0$, so $g=1$, a contradiction. For the pair $(1,2)$ we must have $J=1-2 / v_{1}$. Since $J>0$, we must have $v_{1} \geq 3$ and $J \geq 1 / 3$. As a consequence $N \leq 6(g-1)$.

(2) If $N$ is odd, then $H$ does not have elements of order two. In particular, $k_{2}=0$ and $v_{i}$ must be odd. From the proof of Theorem 2, we only need to take care of case (C.3) with $k_{1}=2$ and $k_{2}=0$. In this case, $0<J=2-2 \Sigma_{i=1}^{2} 1 / v_{i}$ and $3 \leq v_{1} \leq v_{2}$. In this situation we have $J \geq 2 / 3$ and $N \leq 3(g-1)$.

Corollary 5. Under the same hypotheses of Theorem 2.

(i) If $g \not \equiv 1 \bmod 3$, and $N \geq 6(g-1)$, then either $N=6(g-1)$ or $N=8(g-1)$ or $N=12(g-1)$.

(ii) If $g \equiv 1 \bmod 3$, and $N \geq 6(g-1)$, then either $N$ is as in (i) or $N=20(g-1) / 3$. 
Proof. If assume $N>6(g-1)$, then in the proof of Theorem 2 we only need to consider the cases

(a) $\gamma=0, k_{1}=0, k_{2}=4$; and

(b) $\gamma=0, k_{1}=1, k_{2}=2$.

Since we know that $N \geq 8(g-1)$ implies either $N=8(g-1)$ or $N=12(g-1)$, we may assume

$$
6(g-1)<N<8(g-1) .
$$

In case (a), the Riemann-Hurwitz's formula implies $2(g-1)=$ $N\left(2-\Sigma_{i=1}^{4} 1 / w_{j}\right)$. The inequality $(*)$ implies

$$
5 / 3<\Sigma_{j=1}^{4} 1 / w_{j}<7 / 4 .
$$

We may assume $w_{1} \leq w_{2} \leq w_{3} \leq w_{4}$. Then the only 4-tuple $\left(w_{1}, w_{2}, w_{3}, w_{4}\right)$ satisfying the above is $(2,2,2,5)$, in which case, $N=$ $20(g-1) / 3$.

In case (b), the Riemann-Hurwitz's formula asserts $2(g-1)=$ $N\left(2-2 / v_{1}-\Sigma_{j=1}^{2} 1 / w_{j}\right.$, where we may assume $w_{1} \leq w_{2}$. The inequality (*) implies

$$
5 / 3<2 / v_{1}+\Sigma_{j=1}^{2} 1 / w_{j}<7 / 4 .
$$

The only 3 -tuple $\left(v_{1}, w_{1}, w_{2}\right)$ satisfying the above is $(2,2,5)$. Let us consider the surjective homomorphism of section 3

$$
\Phi: \Pi_{1}(X, x) \rightarrow H .
$$

In this case $\Pi_{1}(X, x)$ is generated by $\delta_{1}, \eta_{1}, \theta_{1}$ and $\theta_{2}$ with the only relation $\delta_{1} \eta_{1} \theta_{1} \theta_{2}$. The kernel of the above homomorphism is a normal subgroup containing the elements $\theta_{1}^{2}, \theta_{2}^{5}, \delta_{1}^{2}$ and $\delta_{1} \eta_{1}$. Let us consider the smallest normal subgroup $T$ of $\Pi_{1}(X, x)$ containing the above elements and let us consider the group $\Pi_{1}(X, x) / T$. The order of this group is greater than $N$. It is easy to see that this group is $\mathbf{Z} / 2 \mathbf{Z}$ and, in particular, $N \leq 2$, a contradiction.

Corollary 6. Under the same hypotheses of Theorem 2. If $N>4(g-1)$, then $N=\frac{4 n(g-1)}{(n-2)}$. 
Proof. Let us assume $N>4(g-1)$. From the proof of theorem 2, we only need to consider the following situations:

(1) $\gamma=0, k_{1}=0, k_{2}=4$

(2) $\gamma=0, k_{1}=1, k_{2}=2$.

(3) $\gamma=0, k_{1}=2, k_{2}=0$.

Since we know that $N \geq 6(g-1)$ implies either $N=6(g-1)$, $N=\frac{20(g-1)}{3}($ in which case $g \equiv 1 \bmod 3), N=8(g-1)$ or $N=$ $12(g-1)$, we may, from now on, assume that

$$
4(g-1)<N<6(g-1) .
$$

In case (1), we may assume $w_{1} \leq w_{2} \leq w_{2} \leq w_{3} \leq w_{4}$. The Riemann-Hurwitz's formula implies

$$
2(g-1)=N\left(2-\Sigma_{j=1}^{4} 1 / w_{j}\right) .
$$

The above equality and (*) imply

$$
3 / 2<\Sigma_{j=1}^{4} 1 / w_{j}<5 / 3
$$

The only 4 -tuples $\left(w_{1}, w_{2}, w_{3}, w_{4}\right)$ satisfying the above are $(2,2,2, n)$, $n \geq 7$ and $(2,2,3, k), k \in\{4,5\}$. For the 4 -tuple $(2,2,2, n), n \geq 7$, the Riemann-Hurwitz's formula implies $N=\frac{4 n(g-1)}{(n-2)}$. For the 4-tuple $(2,2,3, k), k \in\{4,5\}$ the Riemann-Hurwitz's formula implies that either $N=\frac{24(g-1)}{5}$ (in which case $g \equiv 1 \bmod 5$ ) or $N=\frac{30(g-1)}{7}$ (in which case $\left.g \equiv 1 \bmod 7\right)$. These two possibles values of $N$ have the desired form.

In case (2), we may assume $w_{1} \leq w_{2}$ The Riemann-Hurwitz's formula implies

$$
2(g-1)=N\left(2-2 / v_{1}-\Sigma_{j=1}^{2} 1 / w_{j}\right)
$$

The above equality and (*) imply

$$
3 / 2<2 / v_{1}+\Sigma_{j=1}^{2} 1 / w_{j}<5 / 3 .
$$


The only 3 -tuples $\left(v_{1}, w_{1}, w_{2}\right)$ satisfying the above are $(2,2, n)$, $n \geq 7$ and $(2,3, k), k \in\{4,5\}$.

For the 3-tuple $(2,2, n), n \geq 7$, the Riemann-Hurwitz's formula implies $N \leq 28(g-1) / 5<4(g-1)$ a contradiction to our assumptions on $N$. For the 3 -tuple $(2,3,4)$, consider the surjective homomorphism of section 3

$$
\Phi: \Pi_{1}(X, x) \rightarrow H .
$$

In this case $\Pi_{1}(X, x)$ is generated by $\delta_{1}, \eta_{1}, \theta_{1}$ and $\theta_{2}$ with the only relation $\delta_{1} \eta_{1} \theta_{1} \theta_{2}$. The kernel of the above homomorphism is a normal subgroup containing the elements $\delta_{1}^{2}, \delta_{1} \eta_{1}, \theta_{1}^{3}$ and $\theta_{2}^{4}$. Consider the smallest normal subgroup $T$ of $\Pi_{1}(X, x)$ containing the above elements and the group $\Pi_{1}(X, x) / T$. The order of this group is greater than $N$. It is easy to see that this group is $\mathbf{Z} / 2 \mathbf{Z}$. Then $N \leq 2$ a contradiction. For the 3 -tuple $(2,3,5)$, consider the surjective homomorphism of section 3

$$
\Phi: \Pi_{1}(X, x) \rightarrow H .
$$

In this case $\Pi_{1}(X, x)$ is generated by $\delta_{1}, \eta_{1}, \theta_{1}$ and $\theta_{2}$ with the only relation $\delta_{1} \eta_{1} \theta_{1} \theta_{2}$. The kernel of the above homomorphism is a normal subgroup containing the elements $\delta_{1}^{2}, \delta_{1} \eta_{1}, \theta_{1}^{3}$ and $\theta_{2}^{5}$. Consider the smallest normal subgroup $T$ of $\Pi_{1}(X, x)$ containing the above elements and the group $\Pi_{1}(X, x) / T$. The order of this group is greater than $N$. It is easy to see that this group is $\mathbf{Z} / 2 \mathbf{Z}$. Then $N \leq 2$ a contradiction. In the case (3), we may assume $v_{1} \leq v_{2}$. The Riemann-Hurwitz's formula implies

$$
2(g-1)=N\left(2-2 \Sigma_{i=1}^{2} 1 / v_{i}\right) .
$$

The above equality and $(*)$ implies $3 / 4<1 / v_{1}+1 / v_{2}<5 / 6$. It is easy to see that there is no pair $\left(v_{1}, v_{2}\right)$ satisfying the above inequalities.

Theorem 3. Let $S$ be a closed Riemann surface of genus $g \geq 2$ and let $H$ be an abelian group of conformal automorphisms of $S$ satisfying condition (A). Then the order of $H$ is at most $2(g+1)$ (resp., 16) if $g \neq 5$ (resp., $g=5$ ). Moreover, the above bounds are the best. In genus 5 there is a group $H$ isomorphic to $\mathbf{Z} / 2 \mathbf{Z} \oplus \mathbf{Z} / 2 \mathbf{Z} \oplus \mathbf{Z} / 2 \mathbf{Z} \oplus$ $\mathbf{Z} / 2 \mathbf{Z}$ satisfying condition ( $A)$, and for every genus there is a group $H$ isomorphic to $\mathbf{Z} / 2 \mathbf{Z} \oplus \mathbf{Z} /(g+1) \mathbf{Z}$ satisfying condition $(A)$. 
Proof. The existence of groups $H$ as in Theorem 3 is done in section 6. Let $S$ of genus $g \geq 2$ and $H$ be as in the hypotheses. Denote by $N$ the order of $H$. The quotient Riemann surface $S / H$ has signature of type

$$
\left(\gamma, 2 k_{1}+k_{2} ; v_{1}, v_{1}, v_{2}, v_{2}, \ldots, v_{k_{1}}, v_{k_{1}}, 2, \ldots, 2\right) .
$$

In this case, the Riemann-Hurwitz's implies

$$
2(g-1)=N\left(2(\gamma-1)+2 \Sigma_{i=1}^{k_{1}}\left(1-1 / v_{i}\right)+k_{2} / 2\right) .
$$

We look for minimal $J$ such that, $0<J=2(\gamma-1)+2 \sum_{i=1}^{k_{1}}(1-$ $\left.\left.1 / v_{i}\right)+k_{2} / 2\right)$

(A) Case $\gamma \geq 2$. In this case, $J \geq 2$ and $N \leq g-1$.

(B) Case $\gamma=1$. In this case, $J=2 \sum_{i=1}^{k_{1}}\left(1-1 / v_{i}\right)+k_{2} / 2$. Since $g \geq 2$, we must have $k_{1}+k_{2}>0$.

(B.1) If $k_{1}>0$, then $J \geq 1$ in which case $N \leq g-1$.

(B.2) If $k_{1}=0$, then $k_{2} \geq 2$. In fact, assume $k_{2}=1$ and consider on the punctured surface $X$ (as defined in section 2) a basis $\alpha, \beta$, $\theta$ (also as defined in section 2). Any simple loop free homotopic to $\theta$ lifts to a path and its square lifts to a loop on the surface $S^{\prime}$ (as defined in section 2). The loop $[\alpha, \beta]$ lifts to a loop (since the group $H$ is abelian) and is free homotopic to $\theta$, contradicting the above. Now, $k_{2} \geq 2$ implies that $J \geq 1$ and $N \leq g-1$.

(C) Case $\gamma=0$. In this case, $J=-2+2 \sum_{i=1}^{k_{1}}\left(1-1 / v_{i}\right)+k_{2} / 2$ and $2 k_{1}+k_{2} \geq 4$.

(C.1) If $2 k_{1}+k_{2} \geq 6$, then $J \geq 1$ and $N \leq 2(g-1)$.

(C.2) If $2 k_{1}+k_{2}=5$, then the only possible pairs $\left(k_{1}, k_{2}\right)$ are given by $(0,5),(1,3)$ and $(2,1)$.

(1) If $k_{1}=0$ and $k_{2}=5$, then $J=5 / 2-2=1 / 2$ and $4(g-1)=$ $N$. In particular, 4 divides $N$. Let us consider the surjective homomorphism of section 3

$$
\Phi: \Pi_{1}(X, x) \rightarrow H
$$


In this case $\Pi_{1}(X, x)$ is generated by $\theta_{j}, j=1, \ldots, 5$, with the only relation $\theta_{1} \theta_{2} \theta_{3} \theta_{4} \theta_{5}=1$. The kernel of the above homomorphism is a normal subgroup containing the elements $\theta_{j}^{2}$ and the commutators of them. Let us consider the smallest normal subgroup $T$ of $\Pi_{1}(X, x)$ containing the above elements and the group (abelian) $\Pi_{1}(X, x) / T$. The order of this group is greater than $N$. This group is $\mathbf{Z} / 2 \mathbf{Z} \oplus \mathbf{Z} / 2 \mathbf{Z} \oplus \mathbf{Z} / 2 \mathbf{Z} \oplus \mathbf{Z} / 2 \mathbf{Z}$ and, in particular, $N \leq 16$. Since 4 divides $N$, we have three possibilities for $N$, that is, either $N=4, N=8$ or $N=16$. In the first case, $g=2, H$ is isomorphic to $\mathbf{Z} / 2 \mathbf{Z} \oplus \mathbf{Z} / 2 \mathbf{Z}$ and we have $N=2(g+1)$. In the case $N=8$, we have $g=3, H$ isomorphic to $\mathbf{Z} / 2 \mathbf{Z} \oplus \mathbf{Z} / 2 \mathbf{Z} \oplus \mathbf{Z} / 2 \mathbf{Z}$ and we have $N=2(g+1)$. In the last case, $N=16$, we have $g=5$ and $H$ is isomorphic to $\Pi_{1}(X, x) / T$.

(2) If $k_{1}=1$ and $k_{2}=3$, then $J=3 / 2-2 / v_{1}$.

(2.1) If $v_{1}=2$, then $J=1 / 2$ and $N=4(g-1)$. Thus 4 divides $N$. Let us consider the surjective homomorphism of section 3

$$
\Phi: \Pi_{1}(X, x) \rightarrow H
$$

In this case $\Pi_{1}(X, x)$ is generated by $\delta_{1}, \eta_{1}, \theta_{j}, j=1,2,3$, with the only relation $\theta_{1} \theta_{2} \theta_{3} \delta_{1} \eta_{1}=1$. The kernel of the above homomorphism is a normal subgroup containing the elements $\theta_{j}^{2}$, $\delta_{1}^{2}, \eta_{1}^{2}, \delta_{1} \eta_{1}$ and the commutators of them. Let us consider the smallest normal subgroup $T$ of $\Pi_{1}(X, x)$ containing the above elements and the group (abelian) $\Pi_{1}(X, x) / T$. The order of this group is greater than $N$. It is easy to see that this group is $\mathbf{Z} / 2 \mathbf{Z} \oplus \mathbf{Z} / 2 \mathbf{Z} \oplus \mathbf{Z} / 2 \mathbf{Z}$ and $N \leq 8$. Since 4 divides $N$, we have two possibilities for $N$, that is, either $N=4$ or $N=8$. In the first case, $g=2$ and $H$ is isomorphic to $\mathbf{Z} / 2 \mathbf{Z} \oplus \mathbf{Z} / 2 \mathbf{Z}$. In the case $N=8, g=3$ and $H$ is isomorphic to $\Pi_{1}(X, x) / T$. Observe that in both cases $N \leq 2(g+1)$

(2.2) If $v_{1}=3$, then $J=5 / 6$ and $5 N=12(g-1)$. In particular, 12 divides $N$. Let us consider the surjective homomorphism of section 3

$$
\Phi: \Pi_{1}(X, x) \rightarrow H
$$


In this case $\Pi_{1}(X, x)$ is generated by $\delta_{1}, \eta_{1}, \theta_{j}, j=1,2,3$, with the only relation $\theta_{1} \theta_{2} \theta_{3} \delta_{1} \eta_{1}=1$. The kernel of the above homomorphism is a normal subgroup containing the elements $\theta_{j}^{2}$, $\delta_{1}^{3}, \eta_{1}^{3}, \delta_{1} \eta_{1}$ and the commutators of them. Let us consider the smallest normal subgroup $T$ of $\Pi_{1}(X, x)$ containing the above elements and the group (abelian) $\Pi_{1}(X, x) / T$. The order of this group is greater than $N$. It is easy to see that this group is $\mathbf{Z} / 2 \mathbf{Z} \oplus \mathbf{Z} / 2 \mathbf{Z} \oplus \mathbf{Z} / 3 \mathbf{Z}$, so $N \leq 12$. Since 12 divides $N$, we must have $N=12$ and $H$ is the above group. In this case $g=6$ and $N=2 g$.

(2.3) If $v_{1} \geq 4$, then $J \geq 1$ and $N \leq 2(g-1)$.

(3) If $k_{1}=2$ and $k_{2}=1$, then $J=5 / 2-2 \Sigma_{i=1}^{2} 1 / v_{i}$. We may assume $2 \leq v_{1} \leq v_{2}$.

(3.1) If $v_{1}=v_{2}=2$, we can use similar arguments as in case $k_{1}=0$ and $k_{2}=5$.

(3.2) If $v_{1}=2$ and $v_{2}=3$, then $J=5 / 6$ and $12(g-1)=$ $5 N$. In particular, 12 divides $N$. Let us consider the surjective homomorphism of section 3

$$
\Phi: \Pi_{1}(X, x) \rightarrow H
$$

In this case $\Pi_{1}(X, x)$ is generated by $\delta_{1}, \eta_{1}, \delta_{2}, \eta_{2}, \theta_{1}$, with the only relation $\theta_{1} \delta_{2} \eta_{2} \delta_{1} \eta_{1}=1$. The kernel of the above homomorphism is a normal subgroup containing the elements $\theta_{1}^{2}, \delta_{1}^{2}$, $\eta_{1}^{2}, \delta_{2}^{3}, \eta_{2}^{3}, \delta_{1} \eta_{1}, \delta_{2} \eta_{2}$ and the commutators of them. Let us consider the smallest normal subgroup $T$ of $\Pi_{1}(X, x)$ containing the above elements and the group (abelian) $\Pi_{1}(X, x) / T$. The order of this group is greater than $N$. It is easy to see that this group is $\mathbf{Z} / 2 \mathbf{Z} \oplus \mathbf{Z} / 3 \mathbf{Z}$. In particular, $N \leq 6$ and since 12 divides $N$, we have a contradiction.

(3.3) If $v_{1} \geq 2$ and $v_{2} \geq 4$, then $J \geq 1$ and $N \leq 2(g-1)$.

(3.4) If $v_{1}=3$ and $v_{2} \geq 3$, then $J \geq 7 / 6$ and $N \leq 12(g-1) / 7 \leq$ $2(g+1)$.

(C.3) If $2 k_{1}+k_{2}=4$, then the only possible pairs $\left(k_{1}, k_{2}\right)$ are given by $(0,4),(1,2)$ or $(2,0)$. 
(1) If $k_{1}=0$ and $k_{2}=4$, then $J=0$; a contradiction.

(2) If $k_{1}=1$ and $k_{2}=2$, then $J=1-2 / v_{1}$. Since $J>0, v_{1} \geq 3$ and $J \geq 1 / 3$. In this case, $N \leq 6(g-1)$.

(3) If $k_{1}=2$ and $k_{2}=0$, then $J=2-2\left(1 / v_{1}+1 / v_{2}\right)$. We may assume that $2 \leq v_{1} \leq v_{2}$. Since $J>0,\left(v_{1}, v_{2}\right) \neq(2,2)$.

(3.1) If $v_{1} \geq 4$, then $J \geq 1$ and $N \leq 2(g-1)$.

(3.2) If $v_{1}=3$ and $v_{2} \geq 6$, then $J>1$ and $N<2(g-1)$.

(3.3) If $v_{1}=3$ and $v_{2}=5$, then $J=14 / 15$ and $15(g-1) / 7=$ $N$. In particular, 15 divides $N$. Let us consider the surjective homomorphism of section 3

$$
\Phi: \Pi_{1}(X, x) \rightarrow H
$$

In this case $\Pi_{1}(X, x)$ is generated by $\delta_{1}, \eta_{1}, \delta_{2}, \eta_{2}$, with the only relation $\delta_{2} \eta_{2} \delta_{1} \eta_{1}=1$. The kernel of the above homomorphism is normal subgroup containing the elements $\delta_{1}^{3}, \eta_{1}^{3}, \delta_{2}^{5}, \eta_{2}^{5}, \delta_{1} \eta_{1}$, $\delta_{2} \eta_{2}$ and the commutators of them. Let us consider the smallest normal subgroup $T$ of $\Pi_{1}(X, x)$ containing the above elements and the group (abelian) $\Pi_{1}(X, x) / T$. The order of this group is greater than $N$. It is easy to see that this group is $\mathbf{Z} / 3 \mathbf{Z} \oplus \mathbf{Z} / 5 \mathbf{Z}$ and, in particular, $N \leq 15$. Since 15 divides $N$, we have $N=15$, $H$ is the above group and $g=8$.

(3.4) If $v_{1}=3$ and $v_{2}=4$, then $J=5 / 6$ and $12(g-1) / 5=$ $N$. In particular, 12 divides $N$. Let us consider the surjective homomorphism of section 3

$$
\Phi: \Pi_{1}(X, x) \rightarrow H
$$

In this case $\Pi_{1}(X, x)$ is generated by $\delta_{1}, \eta_{1}, \delta_{2}, \eta_{2}$, with the only relation $\delta_{2} \eta_{2} \delta_{1} \eta_{1}=1$. The kernel of the above homomorphism is a normal subgroup containing the elements $\delta_{1}^{3}, \eta_{1}^{3}, \delta_{2}^{4}, \eta_{2}^{4}, \delta_{1} \eta_{1}$, $\delta_{2} \eta_{2}$ and the commutators of them. Let us consider the smallest normal subgroup $T$ of $\Pi_{1}(X, x)$ containing the above elements and the group (abelian) $\Pi_{1}(X, x) / T$. The order of this group is greater than $N$. It is easy to see that this group is $\mathbf{Z} / 3 \mathbf{Z} \oplus \mathbf{Z} / 4 \mathbf{Z}$ 
and, in particular, $N \leq 12$. Since 12 divides $N$, we have $N=12$, $H$ is the above group and $g=6$.

(3.5) If $v_{1}=3$ and $v_{2}=3$, then $J=2 / 3$ and $3(g-1)=$ $N$. In particular, 3 divides $N$. Let us consider the surjective homomorphism of section 3

$$
\Phi: \Pi_{1}(X, x) \rightarrow H
$$

In this case $\Pi_{1}(X, x)$ is generated by $\delta_{1}, \eta_{1}, \delta_{2}, \eta_{2}$, with the only relation $\delta_{2} \eta_{2} \delta_{1} \eta_{1}=1$. The kernel of the above homomorphism is a normal subgroup containing the elements $\delta_{1}^{3}, \eta_{1}^{3}, \delta_{2}^{3}, \eta_{2}^{3} \delta_{1} \eta_{1}$, $\delta_{2} \eta_{2}$ and the commutators of them. Let us consider the smallest normal subgroup $T$ of $\Pi_{1}(X, x)$ containing the above elements and the group (abelian) $\Pi_{1}(X, x) / T$. The order of this group is greater than $N$. It is easy to see that this group is $\mathbf{Z} / 3 \mathbf{Z} \oplus \mathbf{Z} / 3 \mathbf{Z}$ and, in particular, $N \leq 9$. Since 3 divides $N$, we have three possibilities for $N$, that is, $N=3, N=6$ or $N=9$. In the first case $g=2$, in the second $g=3$ and in the last $g=4$. Anyway, in the three cases we have $N<2(g+1)$.

(3.6) If $v_{1}=2$ and $v_{2} \geq 3$, then $J=1-2 / v_{2}$ and $N=2 v_{2}(g-$ $1) /\left(v_{2}-2\right)$. Let us consider the surjective homomorphism of section 3

$$
\Phi: \Pi_{1}(X, x) \rightarrow H
$$

In this case $\Pi_{1}(X, x)$ is generated by $\delta_{1}, \eta_{1}, \delta_{2}, \eta_{2}$, with the only relation $\delta_{2} \eta_{2} \delta_{1} \eta_{1}=1$. The kernel of the above homomorphism is a normal subgroup containing the elements $\delta_{1}^{2}, \eta_{1}^{2}, \delta_{2}^{v_{2}}, \eta_{2}^{v_{2}} \delta_{1} \eta_{1}$, $\delta_{2} \eta_{2}$ and the commutators of them. Let us consider the smallest normal subgroup $T$ of $\Pi_{1}(X, x)$ containing the above elements and the group (abelian ) $\Pi_{1}(X, x) / T$. The order of this group is greater than $N$. It is easy to see that this group is $\mathbf{Z} / 2 \mathbf{Z} \oplus \mathbf{Z} / v_{2} \mathbf{Z}$ and, in particular, $N \leq 2 v_{2}$. In this case

$$
2(g+1)=\left(v_{2}-2\right) N / v_{2}+4 \geq N
$$


Remark. Observe from the proof that if $g=5$, then the maximal possible order of an abelian group is 16 in which case, it is isomorphic to the group $\mathbf{Z} / 2 \mathbf{Z} \oplus \mathbf{Z} / 2 \mathbf{Z} \oplus \mathbf{Z} / 2 \mathbf{Z} \oplus \mathbf{Z} / 2 \mathbf{Z}$. If $g \neq 5$, then the maximal order is $2(g+1)$ in which case the group is isomorphic to $\mathbf{Z} / 2 \mathbf{Z} \oplus \mathbf{Z} /(g+1) \mathbf{Z}$. In section 6 we construct explicitly these groups.

Theorem 4. Let $S$ be a closed Riemann surface of genus $g \geq 2$ and let $H$ be a cyclic group of conformal automorphisms of $S$ satisfying condition (A). Then the order of $H$ can be at most $2(g+1)$ (resp., $2 g$ ) if $g$ is even (resp., odd). Moreover, in every even genus $g$ (resp., odd genus) there is a cyclic group $H$ of order $2(g+1)$ (resp., of order $2 g$ ), satisfying condition $(A)$.

Proof. The last part of Theorem 4, that is, the existence of cyclic groups of the desired orders is done in section 6 . If the group is cyclic of order $N$, then the quotient Riemann surface $S / H$ has signature $\left(\gamma, 2 k_{1} ; v_{1}, v_{1}, v_{2}, v_{2}, \ldots, v_{k_{1}}, v_{k_{1}}\right)$, that is $k_{2}=0$. In this case the Riemann-Hurwitz's formula implies the equality

$$
g-1=N\left(\gamma-1+\sum_{i=1}^{k_{1}}\left(1-1 / v_{i}\right)\right)
$$

To find the maximal value for $N$, we need to find the minimal possible value for $J$ such that, $0<J=\gamma-1+\sum_{i=1}^{k_{1}}\left(1-1 / v_{i}\right)$. From now on, we assume $N \geq 2$.

(A) Case $\gamma \geq 2$. In this case, $J \geq 1$ and $N \leq g-1$.

(B) Case $\gamma=1$. In this case, $J=\sum_{i=1}^{k_{1}}\left(1-1 / v_{i}\right)=k_{1}-\sum_{i=1}^{k_{1}} 1 / v_{i} \geq$ $k_{1}-k_{1} / 2=k_{1} / 2 \geq 1 / 2$. Thus, $N \leq 2(g-1)$.

(C) Case $\gamma=0$. In this case, $k_{1} \geq 2$ (by hypotheses) and $J=$ $k_{1}-1-\sum_{i=1}^{k_{1}} 1 / v_{i}$.

(C.1) If $k_{1} \geq 6$, then $J \geq 2$ and $N \leq(g-1) / 2$.

(C.2) If $k_{1}=5$, then $J \geq 3 / 2$ and $N \leq 2(g-1) / 3$. 
(C.3) If $k_{1}=4$, then $J \geq 1$ and $N \leq g-1$.

(C.4) If $k_{1}=3$, then $J \geq 1 / 2$ and $N \leq 2(g-1)$.

(C.5) If $k_{1}=2$, then $J=1-\Sigma_{i=1}^{2} 1 / v_{i}$ and $N=(g-1) / J$. Since $J>0$, we cannot have $v_{1}=v_{2}=2$. We may assume $2 \geq v_{1} \geq v_{2}$.

(1) If $v_{1} \geq 4$, then $J \geq 1 / 2$ and $N \leq 2(g-1)$.

(2) If $v_{1}=3$ and $v_{2} \geq 6$, then $J \geq 1 / 2$ and $N \leq 2(g-1)$.

(3) If $v_{1}=3$ and $v_{2}=5$, then $J=7 / 15$ and $N=15(\mathrm{~g}-$ 1)/7. In particular, $N$ is divisible by 15 . Consider the surjective homomorphism of section 3

$$
\Phi: \Pi_{1}(X, x) \rightarrow H
$$

In this case $\Pi_{1}(X, x)$ is generated by $\delta_{1}, \eta_{1}, \delta_{2}, \eta_{2}$, with the only relation $\delta_{2} \eta_{2} \delta_{1} \eta_{1}=1$. The kernel of the above homomorphism is a normal subgroup containing the elements $\delta_{1}^{3}, \eta_{1}^{3}, \delta_{2}^{5}, \eta_{2}^{5} \delta_{1} \eta_{1}$, $\delta_{2} \eta_{2}$ and the commutators of them. Let us consider the smallest normal subgroup $T$ of $\Pi_{1}(X, x)$ containing the above elements and the group (abelian) $\Pi_{1}(X, x) / T$. The order of this group is greater than $N$. It is easy to see that this group is $\mathbf{Z} / 3 \mathbf{Z} \oplus \mathbf{Z} / 5 \mathbf{Z}$, which is a cyclic group of order 15 and, in particular, $N \leq 15$. Since 15 divides $N$, we must have $N=15$ and the group $H$ is the cyclic group of order 15. In this case $g=8$ and $N<2 g$.

(4) If $v_{1}=3$ and $v_{2}=4$, then $J=5 / 12$ and $N=12(g-$ 1) $/ 5$. In particular, 12 divides $N$. Let us consider the surjective homomorphism of section 3

$$
\Phi: \Pi_{1}(X, x) \rightarrow H
$$

In this case $\Pi_{1}(X, x)$ is generated by $\delta_{1}, \eta_{1}, \delta_{2}, \eta_{2}$, with the only relation $\delta_{2} \eta_{2} \delta_{1} \eta_{1}=1$. The kernel of the above homomorphism is a normal subgroup containing the elements $\delta_{1}^{3}, \eta_{1}^{3}, \delta_{2}^{4}, \eta_{2}^{4} \delta_{1} \eta_{1}$, $\delta_{2} \eta_{2}$ and the commutators of them. Let us consider the smallest normal subgroup $T$ of $\Pi_{1}(X, x)$ containing the above elements and the group (abelian ) $\Pi_{1}(X, x) / T$. The order of this group is 
greater than $N$. It is easy to see that this group is $\mathbf{Z} / 3 \mathbf{Z} \oplus \mathbf{Z} / 4 \mathbf{Z}$, which is isomorphic to the cyclic group of order 12 . Thus, $N=$ 12 and $H$ is isomorphic to $\mathbf{Z} / 12 \mathbf{Z}$. In this case $g=6$ and $N=2 g$.

(5) If $v_{1}=v_{2}=3$, then $J=1 / 3$ and $N=3(g-1)$. Thus, 3 divides $N$. Let us consider the surjective homomorphism of section 3

$$
\Phi: \Pi_{1}(X, x) \rightarrow H
$$

In this case $\Pi_{1}(X, x)$ is generated by $\delta_{1}, \eta_{1}, \delta_{2}, \eta_{2}$, with the only relation $\delta_{2} \eta_{2} \delta_{1} \eta_{1}=1$. The kernel of the above homomorphism is a normal subgroup containing the elements $\delta_{1}^{3}, \eta_{1}^{3}, \delta_{2}^{3}, \eta_{2}^{3} \delta_{1} \eta_{1}$, $\delta_{2} \eta_{2}$ and the commutators of them. Let us consider the smallest normal subgroup $T$ of $\Pi_{1}(X, x)$ containing the above elements and the group (abelian) $\Pi_{1}(X, x) / T$. The order of this group is greater than $N$. It is easy to see that this group is $\mathbf{Z} / 3 \mathbf{Z} \oplus \mathbf{Z} / 3 \mathbf{Z}$. Since $H$ is cyclic, $N \leq 9$ and 3 divides $N$, we have that the only possibility is $N=3$. In that case $H$ is isomorphic to $\mathbf{Z} / 3 \mathbf{Z}$.

(6) If $v_{1}=2$ and $v_{2} \geq 3$, then $J=\left(v_{2}-2\right) / 2 v_{2}, N=2 v_{2}(g-$ $1) /\left(v_{2}-2\right)$. In particular, $v_{2}$ divides $N$. Let us consider the surjective homomorphism of section 3

$$
\Phi: \Pi_{1}(X, x) \rightarrow H
$$

In this case $\Pi_{1}(X, x)$ is generated by $\delta_{1}, \eta_{1}, \delta_{2}, \eta_{2}$, with the only relation $\delta_{2} \eta_{2} \delta_{1} \eta_{1}=1$. The kernel of the above homomorphism is a normal subgroup containing the elements $\delta_{1}^{2}, \eta_{1}^{2}, \delta_{2}^{v_{2}}, \eta_{2}^{v_{2}} \delta_{1} \eta_{1}$, $\delta_{2} \eta_{2}$ and the commutators of them. Let us consider the smallest normal subgroup $T$ of $\Pi_{1}(X, x)$ containing the above elements and the group (abelian) $\Pi_{1}(X, x) / T$. The order of this group is greater than $N$. It is easy to see that this group is $\mathbf{Z} / 2 \mathbf{Z} \oplus \mathbf{Z} / v_{2} \mathbf{Z}$ and, in particular, $N \leq 2 v_{2}$. Since $v_{2}$ divides $N$, we have two possibilities for $N$; either $N=v_{2}$ or $N=2 v_{2}$. In the first case, $g=\left(v_{2}-2\right) / 2+1$ and $N=2 g$. In the second case, $g=v_{2}-1$ and $N=2(g+1)$. 
Observe that for all cases except (C.5 - (6)) we always have $N \leq$ $2 g$. The only case in which we can have $N=2(g+1)$ is in (C.5-(6)) for $N=2 v_{2}$. In that case we must have $H$ isomorphic to $\mathbf{Z} / 2 \mathbf{Z} \oplus \mathbf{Z} / v_{2} \mathbf{Z}$. Since $H$ is cyclic, this only can happen if $v_{2}$ is odd. Then $g=v_{2}-1$ must be even.

\section{Maximal Groups with Condition (A) and Con- dition $\left(A^{*}\right)$.}

\section{Maximal Groups with Condition (A)}

The maximal possible order for a non-triangular group $H$ of conformal automorphisms of a closed Riemann surface $\mathrm{S}$ of genus $g \geq 2$, is $12(\mathrm{~g}-$ 1 ). If we assume that $H$ satisfies condition (A), as a consequence of the proof of Theorem 2, we have that this order can be achieved only if $\gamma=0$ and either

(i) $k_{1}=0, k_{2}=4, w_{1}=w_{2}=w_{3}=2$ and $w_{4}=3$; or

(ii) $k_{1}=1, k_{2}=2, v_{1}=w_{1}=2$ and $w_{2}=3$.

In case (ii) we consider the homomorphism of section 3

$$
\Phi: \Pi_{1}(X, x) \rightarrow H
$$

In this case $\Pi_{1}(X, x)$ is generated by $\delta_{1}, \eta_{1}, \theta_{1}, \theta_{2}$, with the only relation $\delta_{1} \eta_{1} \theta_{1} \theta_{2}=1$. The kernel of the above homomorphism is a normal subgroup containing the elements $\delta_{1}^{2}, \eta_{1}^{2}, \theta_{1}^{2}, \theta_{2}^{3}, \delta_{1} \eta_{1}$ and $\delta_{2} \eta_{2}$. Let us consider the smallest normal subgroup $T$ of $\Pi_{1}(X, x)$ containing the above elements and the group $\Pi_{1}(X, x) / T$. The group $H$ is a surjective image of the above one. It is easy to see that this group is

$$
<A, B, C ; A^{2}=B^{2}=C^{3}=B C=1>=<A ; A^{2}=1>.
$$

In particular, $H$ can have at most order two. In particular, the only possible way to get maximal groups is given by groups of type (i). In that case, using the similar argument as above, they are given as surjective images of the group 


$$
<A, B, C, D ; A^{2}=B^{2}=C^{2}=D^{3}=A B C D=1>\text {. }
$$

The following asserts that there are infinitely many genera for which there is a Riemann surface with a maximal group of conformal automorphisms satisfying the condition (A). The technique used in [12], to obtain the same result for maximal groups of conformal automorphisms, is used to get our result.

Theorem 5. There is an infinite sequence of non-negative integers $g_{i}$, all different and bigger than one, for which there is a closed Riemann surface $S_{i}$ of genus $g_{i}$ and a group $H_{i}$ of conformal automorphisms of $S_{i}$ satisfying the condition $(A)$ and having order $12\left(g_{i}-1\right)$, that is, maximal groups.

The proof of the above is a rapid consequence of the following two lemmas.

Lemma 1. Let $K$ be a Kleinian finite normal extension of a Schottky group $G$ of genus $g$ such that the index $[K: G]=p(g-1)$. If $F$ is a subgroup of $G$ which is normal in $K$ and $[G: F]=r$, then (i) $F$ is a Schottky group of genus $\gamma=r(g-1)+1$, and (ii) $K$ is a Kleinian finite normal extension of $F$ of index $[K: F]=p(\gamma-1)$.

Proof. Since $F$ is a subgroup of a free group, $F$ is also free group. The fact that $[G: F]=r$ implies that $F$ is a free group of rank $\gamma=r(g-1)+1$. The group $G$ being purely loxodromic and Kleinian ensures that $F$ is purely loxodromic and Kleinian. A result of Maskit (see [14]) asserts that $F$ is a Schottky group of genus $\gamma$. The second part is consequence of the equality $[K: F]=[K: G][G: F]=$ $\operatorname{pr}(g-1)=p(\gamma-1)$.

Lemma 2. Let $K$ be a Kleinian finite normal extension of a Schottky group $G$. Then there exist infinitely many subgroup $F_{i} \neq G$ of $G$ of finite (different) index which are normal in $K$.

Proof. Denote by $[G, G]$ the commutator subgroup of $G$ and, for each integer $m \geq 2$, denote by $G_{m}$ the subgroup of $G$ generated by the $m$-powers of the elements of $G$. Since $G$ is normal subgroup of 
$K$, both groups $[G, G]$ and $G_{m}$ are also normal subgroups of $K$. Set $G(m)=G_{m}[G, G]$. The quotient group $G / G(m)$ is a finitely generated abelian group for which every element to the power $m$ is the identity. It follows that $G / G(m)$ is finite abelian group of order $m^{g}$. As a consequence, the group $G(m)$ is the desired group. We also have that $G(m)$ is a Schottky group of genus $m^{g}(g-1)+1$.

Now apply Lemmas 2 and 1, in this order, to obtain the desired result. In section 6 we give examples of maximal groups in genus 2,3 , 5 and 17.

\section{Maximal Groups with Condition (A*)}

As observed from theorem 2, the order of a group $H$, acting on a surface $S$ of genus $g \geq 2$, satisfying condition $\left(\mathrm{A}^{*}\right)$ is bounded by $6(g-$ $1)$. This only happen if $S / H$ is the Riemann sphere with signature $(0,4 ; 2,2,3,3)$.

Theorem 6. If $H$ is a group of order $6(g-1)$ and satisfies condition $\left(A^{*}\right)$, then $H$ is of Schottky type.

Proof. In this case, we have that $S / H$ is a Riemann surface of signature $(0,4 ; 2,2,3,3)$. We draw two pairwise disjoint simple loops $\alpha_{1}$ and $\alpha_{2}$, so that each one bounds a topological disc containing two branched values of same order. Condition $\left(\mathrm{A}^{*}\right)$ asserts that each of these loops lift into exactly $6(g-1)$ loops. From this is simple to see that the lifting of each of the three components of $S / H-\left\{\alpha_{1}, \alpha_{2}\right\}$ consists on genus zero holed surfaces. It follows that we may find a collection of $g$ pairwise disjoint simple loops, homologically independent, inside the liftings of the two above loops. Such a collection determines a Schottky uniformization (up to equivalence) for which the group $H$ lifts.

Explicit examples of maximal groups with condition $\left(\mathrm{A}^{*}\right)$ are given in the next section. 


\section{Explicit examples of Kleinian finite normal ex- tensions of Schottky groups}

In this section, we construct explicit examples of kleinian finite normal extensions of Schottky groups and, in particular, explicit examples of (non-triangular) conformal automorphisms of closed Riemann surfaces satisfying condition $(\mathrm{A})$ and condition $\left(\mathrm{A}^{*}\right)$.

\subsection{Kleinian finite normal extensions of Schottky groups of index $4(\mathrm{~g}+1)$}

Let $g \geq 2$ be fixed and consider Möbius transformations as follows. $W(z)=e^{2 \pi i /(g+1)} z, T(z)=1 / z, J_{0}(z)=((p+1 / p) z-2) /(2 z-(p+$ $1 / p)$ ), where $0<p<1$ and $J_{k}=W^{k} \circ J_{0} \circ W^{-k}$, for $k=1, \ldots, g$. Let $p$ be close enough to 1 such that, the translates of the isometric circle of the involution $J_{0}$ under the non-trivial powers of $W$ are all disjoint. Consider the group $G$ generated by the transformations $A_{k}=J_{0} \circ J_{k}$, $k=1, \ldots, g$. It is clear by the choice of $p$ that $G$ is a Schottky group of genus $g$. Observe that

(1) $W \circ A_{k} \circ W^{-1}$ is either $A_{1}^{-1} \circ A_{k+1}$ or $A_{1}^{-1}$,

(2) $T \circ A_{k} \circ T=A_{g-k+1}$, and

(3) $J_{0} \circ A_{k} \circ J_{0}=A_{k}^{-1}$.

Moreover, every element different from the identity in the (finite) group

$<W, T, J_{0}>$, generated by $W, T$ and $J_{0}$, cannot be in $G$. The group $<W, T, J_{0}>$ is isomorphic to $\mathbf{Z} / 2 \mathbf{Z} \oplus \mathbf{D}_{2(g+1)}$ (the cyclic group in the sum is generated by $J_{0}$ and the Dihedral group is generated by $W$ and $T$ ). The group $K$, generated by $G$ and $\left\langle W, T, J_{0}\right\rangle$, is a finite normal extension of $G$ with index $4(g+1)$.

Observe that, we have constructed a Kleinian finite normal extension $F$ of $G$ with index $2(g+1)$ such that $F / G$ is abelian, where the group $F$ is the group generated by $\mathrm{G}, J_{0}$ and $W$. 


\subsection{Cyclic Kleinian finite normal extensions of Schottky groups of index $2(g+1)$ (resp., $2 \mathrm{~g}$ ) for g even (resp., odd)}

Consider the same Schottky group $G$ as in the first example. The group $K$, in this case, is the group generated by $G$ and the transformations $J_{0}$ and $W$. The group $K$ is a Kleinian finite normal extension of $G$ with index $2(g+1)$. Moreover, $K / G$ is isomorphic to the finite group $\mathbf{Z} / 2 \mathbf{Z} \oplus \mathbf{Z} /(g+1) \mathbf{Z}$. If $g$ is even, then this is a cyclic group of order $2(g+1)$.

For $g$ odd we proceed to construct a Kleinian finite normal extension, as desired, in the following way. Consider Möbius transformations as follows. $L(z)=e^{\pi i / g} z, J_{0}(z)=-z, J_{1}(z)=((p+1 / p) z-$ $2) /(2 z-(p+1 / p))$, where $0<p<1$ and $J_{k}=L^{k-1} \circ J_{1} \circ L^{1-k}$, $k=1, \ldots, g$. Let us consider $p$ close enough to 1 such that, the translates of the isometric circle of $J_{1}$ under the non-trivial powers of $L$ are all disjoint. Let us consider the group $G$ generated by the transformations $A_{k}=J_{0} \circ J_{k}, k=1, \ldots, g$. It is clear, because of the choice of $p$, that $G$ is a Schottky group of genus $g$. Observe that $L \circ A_{k} \circ L^{-1}$ is either $A_{k+1}$ or $A_{1}$ and $J_{0} \circ A_{k} \circ J_{0}$ is $A_{k}^{-1}$. Moreover, $J_{0}=L^{g}$ and the group, generated by $J_{0}$ and $L$, is a cyclic group of order $2 g$. Consider as the group $K$ the group generated by $G, L$ and $J_{0}$. The group $K$ is a Kleinian finite normal extension of $G$ such that $K / G$ is a cyclic group of order $2 g$.

\subsection{Kleinian finite normal extensions of Schottky groups of index 8(g-1) for g odd.}

The following example was constructed in [16] and we recall it now. Let us consider the geometrically finite Kleinian group $K$ which is generated by the transformations

$T(z)=1 / z, \quad W(z)=i z \quad$ and $\quad J(z)=((p+1 / p) z-2) /(2 z-(p+1 / p))$,

where $0<p<1$ is close enough to 1 . The group $K$ is in fact isomorphic to the direct product, amalgamated over $\mathbf{Z} / 2 \mathbf{Z}$, of a dihedral group of order four (the Klein group) and a dihedral group of order 8 . The group $K$ is in fact constructed from Maskit's combination theorems. Write $g=2 q+1$ with $q$ an integer greater or equal to 1 , and 
consider the group $H$ generated by the elements $x, w, j$ and $t$, subject to the relations: $x^{q}=e, w^{4}=e, j^{2}=e, t^{2}=e,(w t)^{2}=e,(j t)^{2}=e$, $w x w^{-1}=x^{-1}, t x=x t, j x j=x^{-1}$ and $j w j=x w$. It is not hard to see that $H$ is a finite group of order $8(g-1)$. The homomorphism $\rho: K \rightarrow H$ defined by $\rho(W)=w, \rho(J)=j$ and $\rho(T)=t$ is surjective since $\rho\left(J \circ W \circ J \circ W^{-1}\right)=x$. Set $G$ the kernel of such a homomorphism. By Maskit's combination theorems, the elliptic elements of $K$ are conjugated to either $W, W^{2}, W^{3}, J, T, W \circ T$ or $J \circ T$. None of these transformations belongs to $G$ and, as a consequence, the group $G$ is torsion-free. Moreover, $G$ is purely loxodromic since $K$ has no parabolic elements (also a direct consequence of the Maskit's combination theorems). Any non-trivial relation on $G$ gives a non-trivial relation in $K$, but the non-trivial relations of $K$ are consequence of the elliptic elements of $K$. The fact that $G$ is torsion-free then ensure that $G$ must be a free group. The region of discontinuity $\Omega$ of $K$ is connected and is the same for $G$. The surface $\Omega / G$ is a finite degree branched covering of the finite Riemann surface $\Omega / K$ and, in particular, $G$ is finitely generated. A theorem of Maskit (see [14]) asserts that $G$ is a Schottky group. The Riemann-Hurwitz's formula (see [4]) gives us that the rank of $G$ is $g$ and, as a consequence, $G$ is a Schottky

group of genus $g$ for which $K$ is a Kleinian finite normal extension of $G$ with index $8(g-1)$.

\subsection{An explicit example in genus 5}

We construct a Kleinian finite normal extension $H$ of a Schottky group $G$ of genus 5 such that, $H / G$ is isomorphic to $\mathbf{Z} / 2 \mathbf{Z} \oplus \mathbf{Z} / 2 \mathbf{Z} \oplus \mathbf{Z} / 2 \mathbf{Z} \oplus$ $\mathbf{Z} / 2 \mathbf{Z}$.

Let us consider the circles $L_{j}, j=1, \ldots, 5$, in the Riemann sphere defined by:

(1) $L_{5}=\{z \in \mathbf{C}:|z|=1\}$;

(2) $L_{1}, L_{2}, L_{3}$ and $L_{4}$ are orthogonal to $L_{5}$;

(3) $L_{1}$ and $L_{2}$ are orthogonal;

(4) $L_{3}$ is disjoint to both $L_{1}$ and $L_{2}$; 
(5) $L_{4}$ is orthogonal to both $L_{3}$ and $L_{1}$; and

(6) $L_{4}$ is disjoint from $L_{2}$.

Let us denote by $\sigma_{j}$ the reflection on the circle $L_{j}$, for each $j=$ $1, \ldots, 5$. We denote by $K$ the group generated by all these reflections. By Klein-Maskit's combination theorems, we have that $K$ is a Kleinian group (with orientation reversing Möbius transformations) with connected region of discontinuity. This group $K$ uniformizes a closed disc with 5 points of order two in the border.

Set $A_{1}=\left(\sigma_{3} \sigma_{1}\right)^{2}, A_{2}=\sigma_{2} A_{1} \sigma_{2}, A_{3}=\left(\sigma_{2} \sigma_{3}\right)^{2}, A_{4}=\left(\sigma_{4} \sigma_{2}\right)^{2}$ and $A_{5}=\left(\sigma_{2} \sigma_{4} \sigma_{3}\right)^{2}$. The group $G$ generated by the transformations $A_{1}$, $A_{2}, A_{3}, A_{4}$ and $A_{5}$ is a Schottky group of genus 5.Simple computations show that $G$ is a normal subgroup of $K$ and that $K / G$ is isomorphic to the group $\mathbf{Z} / 2 \mathbf{Z} \oplus \mathbf{Z} / 2 \mathbf{Z} \oplus \mathbf{Z} / 2 \mathbf{Z} \oplus \mathbf{Z} / 2 \mathbf{Z} \oplus \mathbf{Z} / 2 \mathbf{Z}$. If we denote by $K^{+}$ the index two subgroup of orientation preserving transformations of $K$, then we have that $K^{+} / G$ is isomorphic to $\mathbf{Z} / 2 \mathbf{Z} \oplus \mathbf{Z} / 2 \mathbf{Z} \oplus \mathbf{Z} / 2 \mathbf{Z} \oplus \mathbf{Z} / 2 \mathbf{Z}$.

\subsection{Maximal groups in genus 2 with Condition (A)}

For $g=2$, the maximal possible order is $12(2-1)=12$. Let us consider the Möbius transformations $W(z)=e^{2 \pi i / 3} z, T(z)=1 / z, J_{0}(z)=$ $((p+1 / p) z-2) /(2 z-(p+1 / p))$, with $0<p<1$, and $J_{k}=W^{k} \circ J_{0} \circ W^{-k}$ for $k=1,2$. We choose $p$ close enough to 1 so that, the translates of the isometric circle of $J_{0}$ under the non-trivial powers of $W$ are disjoint. Let $G$ be the group generated by the elements $A=J_{0} \circ J_{1}$ and $B=J_{0} \circ J_{2}$. Our choice of $p$ implies that $G$ is a Schottky group of genus 2, with $A$ and $B$ as free generators. If $K$ is the group generated by $G, T, J_{0}$ and $W$, then $K$ is a Kleinian finite normal extension of $G$. Moreover, the group $K / G$ is isomorphic to $D_{3} \oplus \mathbf{Z} / 2 \mathbf{Z}$, where $D_{3}$ is the Dihedral group of order 6 . The surjective homomorphism from last section (maximal groups) can be seen explicitly as:

$$
\Phi:<A, B, C, D ; A^{2}=B^{2}=C^{2}=D^{3}=A B C D=1>\rightarrow K / G,
$$

with $\Phi(A)=\left[J_{0}\right], \Phi(B)=\left[J_{0} \circ T\right], \Phi(C)=[T \circ W]$ and $\Phi(D)=$ $\left[W^{-1}\right]$, where bracket mean the equivalence class in $K / G$.

The subgroup $G(2)$ (as defined in the proof of Lemma 2) is a Schottky group of genus five for which $K$ is a Kleinian finite normal extension of index $48=12(5-1)$. 


\subsection{Maximal group in genus 3 with Condition (A)}

In this case, the maximal order for a group under consideration is $12(3-1)=24$. Observe that the symmetric group in four letters $\mathcal{S}_{4}=<A, B ; A^{4}=B^{2}=(B \circ A)^{3}=1>$, acts as group of conformal automorphisms of the Riemann sphere, where $A(z)=i z$ and $B(z)=$ $(z+1) /(z-1)$. Let $R$ be the loxodromic transformation $R(z)=\lambda^{2} z$, where $\lambda$ is a non-zero complex number of absolute value different from one. Assume $\lambda>1$, so 0 is the repelling fixed point of $R$. Let $G$ be the group generated by $R, B \circ R \circ B$ and $A \circ B \circ R \circ B \circ A^{-1}$. If the absolute value of $\lambda$ is large enough, then the group $G$ is a Schottky group of genus three with the above generators as free ones. The group $K$ generated by $G, A$ and $B$ is a Kleinian finite normal extension of $G$ such that $K / G$ is isomorphic to $\mathcal{S}_{4}$. We have an explicitly surjective homomorphism

$$
\Phi:<\theta_{1}, \theta_{2}, \theta_{3}, \theta_{4} ; \theta_{1}^{2}=\theta_{2}^{2}=\theta_{3}^{2}=\theta_{4}^{3}=\theta_{1} \theta_{2} \theta_{3} \theta_{4}=1>\longrightarrow \Sigma_{4},
$$

given by $\Phi\left(\theta_{1}\right)=A^{-1} \circ C, \Phi\left(\theta_{2}\right)=C, \Phi\left(\theta_{3}\right)=B$ and $\Phi\left(\theta_{4}\right)=B \circ A$, where $C(z)=B \circ A^{2} \circ B(z)=1 / z$.

The subgroup $G(2)$ (as defined in the proof of Lemma 2) is a Schottky group of genus 17 for which $K$ is a Kleinian finite normal extension of index $192=12(17-1)$.

\subsection{Maximal groups with Condition $\left(\mathrm{A}^{*}\right)$}

Let us consider a Kleinian group $K$ obtained as free product of a cyclic group of order two $\left\langle X: X^{2}=1\right\rangle$ and a cyclic one of order three $\left\langle Y: Y^{3}=1\right\rangle$. Let $G_{1}$ be the normalizer of $(Y X)^{2}$ in $K, G_{2}$ the normalizer of $(Y X)^{3}$, and $G_{3}$ the normalizer of $(Y X)^{4}$. Then each of this groups is a Schottky group of genus 2, 3 and 5, respectively. We have that $K / G_{1}, K / G_{2}$ and $K / G_{3}$ are isomorphic to $D_{3}, \mathcal{A}_{4}$ and $\mathcal{S}_{4}$, respectively.

\section{Connection with Handlebodies}

In this section, we apply the results of the above sections to obtain bounds on the orders of finite groups of orientation-preserving homeo- 
morphisms of handlebodies of genus $g \geq 2$. Bounds, for such a groups, are in fact obtained in [15] and [16] using graphs of finite groups.

Let $V_{g}$ be a handlebody of genus $g$ and let $H$ be a finite group of orientation preserving homeomorphisms of $V_{g}$ of order $N$. This group acts as a finite group of orientation preserving homeomorphisms on the boundary $S$ of $V_{g}$, which is a closed orientable surface of genus $g$. It is known [11] that $S$ admits a Riemann surface structure for which $H$ is a group of conformal automorphisms. If we choose $g$ disjoint, homologically independent simple loops on $S$ such that, they are homotopically trivial in $V_{g}$, then the Schottky group (up to conjugation) $G$ determined by these loops satisfies the property that $H$ lifts as a group of conformal automorphisms of its region of discontinuity. In particular, the group $H$ satisfies condition (A) and they have signature different from $(0,3 ; p, q, r)$. As a consequence of the results of the above sections, we obtain the known bounds of [15] and [16].

Theorem 7. Let $H$ be a finite group of orientation preserving homeomorphisms of a handlebody $V_{g}$ of genus $g \geq 2$. Then the order $N$ of $H$ is at most $12(g-1)$. Moreover, if we assume $N \geq 6(g-1)$, then

$$
N \in\{6(g-1), 8(g-1), 12(g-1), 20(g-1) / 3\} .
$$

Theorem 8. Let $H$ be a finite group, of order $N$, of orientation preserving homeomorphisms of a handlebody $V_{g}$ of genus $g \geq 2$. If $N \geq 4(g-1)$, then $N=4 n(g-1) /(n-2)$ for some $n \geq 3$.

Remark. Since we know that every handlebody of genus $g \geq 2$ has a group of orientation preserving homeomorphisms of order $4(g+1)>$ $4(g-1)$, the maximal order for such a group of orientation-preserving homeomorphisms must have the form in Theorem 6. Moreover, for $g$ odd we know that the maximal order is either $12(g-1)$ or $8(g-1)$, that is, $[4 n /(n-2)](g-1)$, where $n \in\{3,4\}$.

Theorem 9. Let $H$ be a finite abelian group of orientation preserving homeomorphisms of a handlebody $V_{g}$ of genus $g \geq 2$. Then the order of $H$ is at most $2(g+1)$ if $g \neq 5$ and at most 16 if $g=5$. Moreover, 
these bounds are achieved by a group isomorphic to $\mathbf{Z} / 2 \mathbf{Z} \oplus \mathbf{Z} /(g+1) \mathbf{Z}$ for $g \neq 5$ and by $\mathbf{Z} / 2 \mathbf{Z} \oplus \mathbf{Z} / 2 \mathbf{Z} \oplus \mathbf{Z} / 2 \mathbf{Z} \oplus \mathbf{Z} / 2 \mathbf{Z}$ for $g=5$.

Theorem 9. Let $H$ be a finite cyclic group of orientation preserving homeomorphisms of a handlebody $V_{g}$ of genus $g \geq 2$. Then the order of $H$ is at most $2(g+1)$ for $g$ even and at most $2 g$ for $g$ odd. These bounds are achieved in every genus.

\section{References}

[1] L. V. Ahlfors and L. Sario. Riemann surfaces. Princeton Univ. Press. Princeton, New Jersey, (1960).

[2] L. Bers. Automorphic forms for Schottky groups. Adv. in Math., 16, pp. 332-361, (1975).

[3] V. Chuckrow. On Schottky groups with applications to Kleinian groups. Ann. of Math. 88, pp. 47-61, (1968).

[4] H. Farkas and I. Kra. Riemann surfaces. Springer - Verlag, New York, (1980).

[5] L. Keen, B. Maskit and C. Series. Geometric finiteness and uniqueness for Kleinian groups with circle packing limit sets. J. reine angew. Math. 436, pp. 209-219, (1993).

[6] V. González and R. Rodríguez. On automorphisms of compact Riemann surfaces of genus four. Complex geometry seminar, volume II, UTFSM, (1986).

[7] R. Hidalgo. Schottky Uniformizations of closed Riemann surfaces with Abelian groups of conformal automorphisms. Glasgow Math. J. bf 35, pp. 17-32, (1993)..

[8] R. Hidalgo. On Schottky groups with automorphisms. Theses Ph. D. of Mathematics, S.U.N.Y. at Stony Brook, (1991). (to be published on Ann. Acad. Sci. Fenn.). 
[9] R. Hidalgo. Dihedral groups are of Schottky type. Preprint.

[10] R. Hidalgo. $\mathcal{A}_{4}, \mathcal{A}_{5}$ and $\mathcal{S}_{4}$ of Schottky type. Preprint.

[11] S. Kerckhoff. The Nielsen realization problem. Annals of Mathematics, 117, pp. 235-265, (1983).

[12] A.M. Macbeath. On a theorem of Hurwitz. Proc. Glasgow Math. Assoc., 5, pp. 90-96, (1961).

[13] B. Maskit. Kleinian groups. Grundlehren der Mathematischen Wissenschaften, volume 287, Springer - Verlag, Berlin, Heildelberg, New York, (1988).

[14] B. Maskit. A characterization of Schottky groups. J. d'Analyse Math., 19, pp. 227-230, (1967).

[15] D. McCullough, A. Miller and B. Zimmermann. Group actions on Handlebodies. Proc. London Math. Soc., 59, pp. 373-416, (1989).

[16] A. Miller and B. Zimmermann. Large groups of symmetries of handlebodies. Proc. Amer. Math. Soc., 106, pp. 829-838, (1989).

[17] K. Nakagawa. On the orders of automorphisms of a closed Riemann surface. Pacific J. of Math., 115, (1984).

[18] R. Ruedy. Symmetric embeddings of Riemann surfaces. In Discontinuous groups and Riemann surfaces. Ed., by Leon Greenberg. Annals of Math. Studies. Princeton Univ. Press., number 79, (1974).

[19] B. Zimmermann. Über Homöomorphismen n-dimensionaler Henkelkörper und endliche Erweiterungen von Schottky-Gruppen. Comment. Math. Helv., 56, pp. 474-486, (1981).

Received : May, 2000. 


\section{RUBEN A. HIDALGO}

Departamento de Matemática

Universidad Técnica Federico Santa María

Casilla 110-V

Valparaíso

Chile

E-Mail: rhidalgo@mat.utfsm.cl 